\title{
La enseñanza de la Semiología. Presentación del texto de Semiología de A. Goic, G. Chamorro y H. Reyes
}

\section{Presentation of the third edition of a text book on medical history taking and physical examination, by A. Goic, G. Chamorro and H. Reyes (Editors)}

Clinicians know that the clinical encounter with the patient constitutes the foremost aspect in diagnosis and therapeutics. We also remember that our first contact with clinical practice was to learn how to perform a good clinical history and thorough physical examination. Even after years of practice we are still learning how to listen to the patient, see, palpate and auscultate but most importantly, to approach a patient with warmth and kindness to unveil the mysteries of diseases and ailments. Books will not teach us these skills and practice is the basis of this learning process, but the text book by Drs. Goic, Chamorro and Reyes is of great help and orientation, when we drift through the different chapters devoted to medical history taking, physical signs, syndromes and laboratory tests. This third edition includes the late Dr. Chamorro as co-editor, because outstanding people do not pass, only transcend. Medical students will enjoy this book and clinicians will also appreciate it, since we are all aware that we never stop learning until we pass... or transcend.

(Rev Med Chile 2011; 139: 119-122).

Key words: Medical history taking; Physical examination; Text books.
'Profesor de Medicina, Universidad de Chile. Miembro de Número, Academia Chilena de Medicina.
I

a Semiología es la rama de la medicina que se ocupa de la identificación de las diversas $\checkmark$ manifestaciones de enfermedad, de cómo buscarlas (semiotécnica) y cómo interpretarlas (clínica semiológica).

La Universidad de Chile inició una Cátedra de Semiología en 1931 y ha habido distinguidísimos profesores de esa asignatura. El Dr. Hernán Alessandri fue profesor de ella a partir de 1937 y fueron profesores el para mi inolvidable Dr. José Manuel Balmaceda, que fue mi profesor y cuya personalidad me dejó una marca para siempre, los Drs. Domingo Urrutia, Miguel Hermosilla, Raúl Yazigi, Jorge Jalil y otros. Ha estado la Semiología siempre inserta en la Medicina Interna, sea como cátedra en si pero dentro de un Servicio de Medicina o como una asignatura dentro de un Departamento docente de Medicina.

La Semiología es la base de la medicina clínica y en verdad es el cimiento sobre el que se construye el trabajo de curar pacientes. Las personas sienten síntomas y presentan signos objetivos, pero hay que saber purificar la sintomatología y los hallazgos del examen para acceder al diagnóstico y al tratamiento adecuados. Los pacientes en general no saben cual es la causa de las molestias que presentan ni saben qué es y qué no es importante de su sintomatología. El médico debe develar la causa y valorar la importancia de cada síntoma. Comúnmente, ni los síntomas ni los signos afloran espontáneamente, hay que buscarlos e interpretarlos. Esa búsqueda e interpretación es la que hace la Semiología. 
Así, la Semiología es lo primero que se ha de enseñar dentro de la enseñanza de la clínica. El alumno deberá aprender a interrogar al enfermo, con mucho tino para no sugerirle las respuestas ni omitir información. Durante esa asignatura el estudiante debe aprender lo importante que es la silla al lado de la cama del paciente, para poder dialogar y obtener la información que no surge cuando hay prisa. En la asignatura de Semiología el alumno deberá aprender que el examen físico debe ser completo y prolijo.

Desde temprano deberá compenetrarse que es tan fácil como grave equivocarse en Medicina y que ello puede evitarse si la búsqueda de síntomas y signos se realiza con minuciosidad. Son muchos los errores que se podrían haber evitado si se hubiese hecho un buen interrogatorio y un examen físico prolijo. Es todo un arte interrogar y examinar a los pacientes.

Es, también, durante la enseñanza de la Semiología, la primera oportunidad para crear en el alumno normas de conducta y disciplina que permitan una relación de respeto mutuo con el paciente, lo que es indispensable en el trabajo clínico. Es claro que la asignatura de Semiología es el momento para enseñar a los estudiantes los síntomas y signos que ocurren habitualmente en las patologías más frecuentes; pero es claro también que es la oportunidad de impartir otras enseñanzas como son esas normas de conducta.

En este período el tutor al lado del alumno tiene un papel insubstituible; él tendrá que enseñar a interrogar y a examinar; a valorar el respeto por la persona enferma y la importancia de la prolijidad; a desconfiar de la debilidad de los conocimientos que disponemos y a enriquecerlos mediante la disciplina de la educación continua. Tiene el tutor el deber de enseñar sobre la importancia de la vestimenta, del lenguaje, de la actitud en general para ganarse el respeto y la confianza del paciente para poder hacer un trabajo con empatía. La buena semiología se logra cuando el médico aporta al paciente tiempo y conocimientos y logra su confianza.

No obstante estar ubicada la Semiología tempranamente en el currículo y ser considerada un cimiento de la práctica clínica, ella nunca se termina de aprender. Cuanta más patología se conoce y más experiencia se tiene, más se enriquece el universo de síntomas y signos que hay que buscar. Además, como todo, la Semiología se olvida y hay que refrescarla. También, la experiencia clínica nos enseña permanentemente a mejor interrogar, a saber buscar lo importante, a aprender a qué exámenes complementarios nos conviene recurrir en cada oportunidad. La buena Semiología, más que saber mucho de algo, es un estilo de ejercer la profesión, una forma de actuar con eficacia y que alcanza a todos los médicos, cualquiera sea la disciplina que cultiven.

Durante la enseñanza de la Semiología se viven días preciosos. De una parte los alumnos están muy motivados por estar iniciándose en la clínica -que es lo que buscaban al entrar a la Escuela de Medicina- y, de otra parte, preciosos porque se enseñan normas de conductas y estilos de trabajo que durarán para siempre.

Ha aparecido la Tercera Edición del texto "Semiología Médica", de los Drs. Alejandro Goic Goic, Gastón Chamorro Zapata y Humberto Reyes Budelovsky ${ }^{1}$. No es el único ni ha sido el primer libro de Semiología que ha aparecido en este país. Recuerdo al menos otros cuatro, los que por lo demás están citados en la bibliografía que trae este texto. Pero ciertamente que éste es el libro de Semiología que ha tenido más trayectoria. Su primera edición ocurrió en 1987, siendo sus autores los Drs. Alejandro Goic G. y Gastón Chamorro Z., el que fue reimpreso los años 1991, 1993, 1994, 1996 y 1997. Luego, en 1998 hubo una segunda edición, en la que se agregó como autor al Dr. Humberto Reyes B. y se mantuvo como autor al Dr. Gastón Chamorro, no obstante que había fallecido poco antes de la publicación.

En esta tercera edición que hoy se presenta, se mantienen los mismos tres autores, a pesar de que el Dr. Chamorro falleció hace más de diez años. Los otros autores han decidido mantener su nombre porque los Capítulos que él escribió persisten vigentes y fue necesario hacerles muy pocas modificaciones. Es claro que se ha deseado mantener al Dr. Chamorro porque él dejó una huella imborrable en muchas generaciones de médicos: notable clínico, muy buen semiólogo, docente de excepción, modesto como pocos, caballeroso y respetuoso con todo el mundo y con una vasta cultura, especialmente musical.

En el historial de este libro, además de tres ediciones y cinco reimpresiones, cabe agregar que fue incorporado al programa de textos de la Oficina Sanitaria Panamericana. De manera que estamos asistiendo ahora a la continuación de un texto de 
estudios que ya han gozado miles de estudiantes de medicina de las Américas, a lo largo de 22 años.

La tenacidad de los autores y el entusiasmo en la mantención de esta obra probablemente se enraízan en la memoria de su maestro, el Dr. Hernán Alessandri. El fue un muy destacado clínico, con poderoso liderazgo en su época, formador de muchísimos médicos entre los que se incluyen los autores de esta obra y no sólo un muy buen semiólogo sino que Profesor de Semiología a partir de 1937 y luego Profesor de Medicina. Ya en el prólogo de la primera edición se percibe como los autores tienen un recuerdo cariñoso y sienten mucha gratitud y profunda admiración hacia el Dr. Alessandri. Pero la nostalgia va más allá de la persona del Dr. Alessandri: alcanza al grupo de médicos que trabajaron junto a ese maestro. Casi todos los autores de capítulos son de ese grupo y se ha conservado como autores a médicos que fueron de esa época pero que ya han fallecido o que están fuera del ejercicio de la docencia y de la profesión por el implacable devenir que con los años nos espera a todas las personas. Da la impresión que los Drs. Goic y Reyes, con melancolía, se resisten a que el tiempo desarme ese grupo en el que se formaron y trabajaron por tanto tiempo.

Este libro tiene una presentación impecable, es ameno, fácil de leer, tiene un nivel de excelencia a lo largo de todos sus capítulos. Facilitan el aprendizaje las numerosas tablas y figuras. De hecho, en sus 770 páginas hay 203 tablas, 346 figuras y 38 láminas a color que facilitan la enseñanza que se desea impartir.

A medida que se va revisando el libro aparecen rincones en él que a uno lo retienen por tener algún atractivo especial. Por ejemplo:

- Los síndromes neurológicos están tratados con sencillez y en forma muy didáctica, aptos para estudiantes que se están iniciando en medicina. Me recordó que en nuestra época de estudiantes, el tomo de neurología del libro de Semiología de los profesores argentinos Padilla y Cossio nos siguió acompañando cuando estudiábamos clínica neurológica en postitulo

- Hay capítulos que muy acertadamente tienen un rico contenido fisiopatológico, que sin duda excede a lo que corresponde a un texto de Semiología y que, al leerlos, me hicieron pensar que podría ser interesante una integración plena de las asignaturas de Fisiopatología y Semiología que enseñamos tan separadamente; quizás manejarlas integradamente contribuiría por una parte a una "basificación" de la clínica, lo que siempre es bueno y por otra, a una fisiopatología más aplicada y próxima a la clínica; Hay un excelente capítulo de anamnesis y examen físico del adulto mayor, que tiene el interés de referirse a un sector de la población cada vez más numeroso y especialmente vulnerable; es cierto que está orientado fuertemente a la evaluación neuropsiquiátrica del paciente y que en éstos a menudo se dan desórdenes de otras áreas;

- Una parte del libro, que representa alrededor de un quinto del texto, está dedicada a exámenes complementarios y ahí se trata el laboratorio clínico corriente, las radiografías de tórax y abdomen, las endoscopias y radiologías digestivas, la ecotomografía de abdomen, la densitometría ósea, los marcadores tumorales, etc. Estos capítulos muestran el convencimiento de los autores que es parte de la semiología la exploración con exámenes complementarios y que la anamnesis y el examen físico bien hechos, orientan hacia qué exámenes hay que pedir y cómo interpretarlos y que éstos, bien usados, son un tremendo regalo de la medicina actual.

- Un capítulo se refiere al Juramento Hipocrático y me pregunté al verlo qué tiene que hacer ésto en un texto de Semiología; luego caí en la cuenta que tiene la más plena cabida por ser el primer texto de enseñanza de clínica que tendrán los estudiantes, pues -aunque no recuerdo que en otros textos de medicina se de un espacio a un código de ética- hoy día es impensable aprender clínica sin impregnarla de ética.

- Un glosario de síntomas y signos y otro de enfermedades figuran en el apéndice y son notables. El glosario de síntomas y signos incluye casi trescientos de ellos, los que son definidos en pocas palabras. Obviamente esta es una valiosísima ayuda para los estudiantes, los que al momento de cursar Semiología tienen que ir introduciéndose en el complicado mundo de las formas de manifestarse de las enfermedades y en el no menos complicado lenguaje médico. El glosario de enfermedades describe somera pero suficientemente algo más de 200 afecciones, lo que obviamente será de mucha utilidad para los estudiantes que se están iniciando en la 
clínica. Fuera de dudas está que estos glosarios de síntomas y enfermedades, especialmente este último, nos pueden ser útiles aún a quienes llevamos muchos años en el ejercicio de esta profesión.

Este texto es un valioso aporte a la enseñanza de la medicina, muy necesario para los estudiantes que se inician en el sagrado oficio de curar enfermos. Ojalá el libro llegue a la casa de todos ellos y no falte en las bibliotecas de las numerosas Escuelas de Medicina.
Es justo agradecer a los autores de esta obra por la contribución que este libro representa a la enseñanza médica y por el esfuerzo y tiempo invertidos en este trabajo.

\section{Referencias}

1. Goic A, Chamorro G, Reyes H. (Editores). Semiología Médica (3a Edición). 2010. Editorial Mediterráneo, Ltda. Santiago de Chile. 